\title{
Two-particle states in the Hubbard model
}

\author{
Manuel Valiente and David Petrosyan \\ Institute of Electronic Structure \& Laser, FORTH, 71110 Heraklion, Crete, Greece
}

(Dated: January 25, 2018)

\begin{abstract}
We consider a pair of bosonic particles in a one-dimensional tight-binding periodic potential described by the Hubbard model with attractive or repulsive on-site interaction. We derive explicit analytic expressions for the two-particle states, which can be classified as (i) scattering states of asymptotically free particles, and (ii) interaction-bound dimer states. Our results provide a very transparent framework to understand the properties of interacting pairs of particles in a lattice.
\end{abstract}

PACS numbers: 03.75.Lm, 37.10.Jk, 03.65.Ge

Many-body quantum systems in spatially-periodic potentials were studied since the early days of quantum theory [1]. Several simple but powerful models have been invented and successfully employed for the description of various condensed matter systems, including the Heisenberg spin and Hubbard models. Lately, the Hubbard model has been attracting renewed attention prompted by the remarkable experimental progress in cooling and trapping bosonic and fermionic atoms in optical lattices [2]. The relevant parameters of this system can be controlled with high precision and can be tuned to implement the Hubbard model with unprecedented accuracy [3].

Recently, Winkler et al. [4] have observed a remarkable Hubbard model phenomenon: A repulsive on-site interaction between a pair of bosonic atoms in an optical lattice can bind them together into an effective, dynamically stable "dimer" [5, 6, 7]. Here we revisit the problem of two bosonic particles in a 1D (one-dimensional) periodic potential described by the Hubbard model. We consider both cases of attractive and repulsive on-site interactions between the particles and derive simple, exact analytic solutions for the two-particle problem. One type of these solutions corresponds to the scattering states of asymptotically free particles, while the other describes the interaction-bound dimer states with energies below or above the energy band of the scattering states [5].

We note that the solution of the Schrödinger equation for the two-particle problem in a lattice can be derived using the Green function approach [4, 5], which although being more general, requires laborious calculations often accompanied by approximations for obtaining explicit analytic results. In contrast, in what follows we give an extremely simple and straightforward derivation of the energies and exact analytic wavefunctions for the two-particles states in a lattice.

The Hamiltonian of the problem is given by

$$
H=-J \sum_{j}\left(b_{j}^{\dagger} b_{j+1}+b_{j+1}^{\dagger} b_{j}\right)+\frac{U}{2} \sum_{j} \hat{n}_{j}\left(\hat{n}_{j}-1\right)
$$

where $b_{j}^{\dagger}\left(b_{j}\right)$ is the bosonic creation (annihilation) operator and $\hat{n}_{j}=b_{j}^{\dagger} b_{j}$ the number operator for site $j$, $J(>0)$ is the tunnel coupling between adjacent sites, and $U$ is the on-site interaction. A standard basis $\left\{\left|n_{j}\right\rangle\right\}$ for Hamiltonian (1) is composed of the eigenstates $\left|n_{j}\right\rangle \equiv \frac{1}{\sqrt{n !}}\left(b_{j}^{\dagger}\right)^{n}|\mathrm{vac}\rangle$ of operator $\hat{n}_{j}$ whose eigenvalues $n=0,1,2, \ldots$ denote the number of bosonic particles at site $j$, and $|\mathrm{vac}\rangle \equiv\left|\left\{0_{j}\right\}\right\rangle$ is the vacuum state.

For a single particle in the periodic potential, the last term on the right-hand side of Eq. (1) does not play a role. The Hubbard Hamiltonian then takes the form

$$
H^{(1)}=-J \sum_{j}\left(\left|x_{j}\right\rangle\left\langle x_{j+1}|+| x_{j+1}\right\rangle\left\langle x_{j}\right|\right),
$$

where $\left|x_{j}\right\rangle=\left|1_{j}\right\rangle$ denotes the state with a single particle at position $x_{j} \equiv d j$ of the $j$ th lattice site, with $d$ the lattice constant. Expanding the single-particle state vector as $|\psi\rangle=\sum_{j} \psi\left(x_{j}\right)\left|x_{j}\right\rangle$, the stationary Schrödinger equation $H^{(1)}|\psi\rangle=E^{(1)}|\psi\rangle$ reduces to the difference equation

$$
-J\left[\psi\left(x_{j-1}\right)+\psi\left(x_{j+1}\right)\right]=E^{(1)} \psi\left(x_{j}\right)
$$

for the wavefunction $\psi\left(x_{j}\right)$. Taking $\psi(x)=\psi_{q}(x) \equiv$ $\exp (i q x)$ immediately yields the well-known result

$$
-2 J \cos (q d) \psi_{q}\left(x_{j}\right)=E_{q}^{(1)} \psi_{q}\left(x_{j}\right)
$$

which implies that the discrete plane waves $\psi_{q}\left(x_{j}\right)=$ $\exp (i q d j)$ are the eigenfunctions of Hamiltonian (2), with the corresponding eigenenergies $E_{q}^{(1)}=-2 J \cos (q d)$, forming a Bloch band of width $4 J$ [1]. The effective mass $m^{*}$ of the particle with quasi-momentum $q$ close to 0 is then given by the usual expression

$$
m^{*}=\hbar^{2}\left[\frac{\partial^{2} E_{q}^{(1)}}{\partial q^{2}}\right]_{q=0}^{-1}=\frac{\hbar^{2}}{2 J d^{2}}
$$

For two particles in a one-dimensional lattice, the Hubbard Hamiltonian can be recast in the explicit form

$$
\begin{aligned}
H^{(2)}= & -J \sum_{j}\left(\left|x_{j}\right\rangle\left\langle x_{j+1}|+| x_{j+1}\right\rangle\left\langle x_{j}\right|\right) \\
& -J \sum_{j}\left(\left|y_{j}\right\rangle\left\langle y_{j+1}|+| y_{j+1}\right\rangle\left\langle y_{j}\right|\right) \\
& +U \sum_{j}\left|x_{j}, y_{j}\right\rangle\left\langle x_{j}, y_{j}\right|
\end{aligned}
$$


while the two-particle state vector can be expanded in terms of the non-symmetrized basis $\left\{\left|x_{j}, y_{j^{\prime}}\right\rangle\right\}$ as

$$
|\Psi\rangle=\sum_{j, j^{\prime}} \Psi\left(x_{j}, y_{j^{\prime}}\right)\left|x_{j}, y_{j^{\prime}}\right\rangle
$$

where $y_{j} \equiv d j$. Clearly, the standard (symmetrized) bosonic basis is related to the non-symmetrized basis via the transformation $\left|2_{j}\right\rangle=\left|x_{j}, y_{j}\right\rangle$ and $\left|1_{j}, 1_{j^{\prime}}\right\rangle=$ $\frac{1}{\sqrt{2}}\left(\left|x_{j}, y_{j^{\prime}}\right\rangle+\left|y_{j}, x_{j^{\prime}}\right\rangle\right)\left(j \neq j^{\prime}\right)$. The stationary Schrödinger equation $H^{(2)}|\Psi\rangle=E^{(2)}|\Psi\rangle$ is now equivalent to the recurrence relation

$$
\begin{aligned}
& -J\left[\Psi\left(x_{j-1}, y_{j^{\prime}}\right)+\Psi\left(x_{j+1}, y_{j^{\prime}}\right)\right. \\
& \left.\quad+\Psi\left(x_{j}, y_{j^{\prime}-1}\right)+\Psi\left(x_{j}, y_{j^{\prime}+1}\right)\right] \\
& \quad+U \delta_{j j^{\prime}} \Psi\left(x_{j}, y_{j^{\prime}}\right)=E^{(2)} \Psi\left(x_{j}, y_{j^{\prime}}\right) .
\end{aligned}
$$

Define the center of mass $R=\frac{1}{2}(x+y)$ and relative $r=x-y$ coordinates. In terms of the new variables, the two-particle wavefunction can be separated as

$$
\Psi(x, y)=e^{i K R} \psi_{K}(r)
$$

where the relative coordinate wavefunction $\psi_{K}(r)$ depends now on the center-of-mass quasi-momentum $K \in$ $[-\pi / d, \pi / d]$ as a continuous parameter. Equation (8) then yields

$$
\begin{aligned}
-J_{K}\left[\psi_{K}\left(r_{i-1}\right)+\psi_{K}\left(r_{i+1}\right)\right]+ & U \delta_{r 0} \psi_{K}\left(r_{i}\right) \\
& =E_{K}^{(2)} \psi_{K}\left(r_{i}\right),
\end{aligned}
$$

with $J_{K} \equiv 2 J \cos (K d / 2)$ and $r_{i}=d i\left(i=j-j^{\prime}\right)$.

(i) Consider first the scattering solutions for a pair of asymptotically free particles [4, 5]. Since the action of the short-range scattering potential $U \delta_{r 0}$ amounts to a unitary phase-shift (see below), the spectrum of such solutions is given by the sum of the spectra for two free particles $x$ and $y$ with momenta $q_{x}=K / 2+k$ and $q_{y}=K / 2-k$ :

$$
\begin{aligned}
E_{K, k}^{(2)}=E_{q_{x}}^{(1)}+E_{q_{y}}^{(1)} & =-4 J \cos (K d / 2) \cos (k d) \\
& =-2 J_{K} \cos (k d)
\end{aligned}
$$

Obviously, this result also follows directly from Eq. (10), with $U=0$, upon substitution of the plane waves $\psi_{K}(r)=\psi_{K, k}^{0}(r)=\exp ( \pm i k r)$ [5]. For a given value of the center-of-mass momentum $K$, and thereby $J_{K}$, the lowest $E_{K, 0}^{(2)}=-2 J_{K}$ and highest $E_{K, \pi}^{(2)}=2 J_{K}$ energy states of a pair of asymptotically free particles correspond, respectively, to the relative momenta $k \rightarrow 0$ and $k \rightarrow \pi / d$. The energy spectrum of Eq. (11), and the corresponding density of states

$$
\rho(E, K)=\frac{L}{2 \pi} \frac{\partial k}{\partial E}=\frac{L}{2 \pi d} \frac{1}{\sqrt{[4 J \cos (K d / 2)]^{2}-E^{2}}},
$$

with $L$ a quantization length, are shown in Fig. 1.
We can now derive an explicit expression for the wavefunction of the scattering states. Substituting $E_{K, k}^{(2)}$ of (11) into Eq. (10), setting $\psi_{K, k}(0)=C$ and using the bosonic symmetry $\psi_{K, k}(r)=\psi_{K, k}(-r)$, after little algebra we obtain

$$
\psi_{K, k}\left(r_{i}\right)=C \cos \left(k r_{i}\right)+C \frac{U \csc (k d)}{2 J_{K}} \sin \left(k\left|r_{i}\right|\right) .
$$

In terms of the plane waves undergoing the scattering phase shift $\delta_{K, k}$, the wavefunction reads

$$
\begin{aligned}
\psi_{K, k}\left(r_{i}\right) & =e^{i k\left|r_{i}\right|} e^{i \delta_{K, k}}+e^{-i k\left|r_{i}\right|} e^{-i \delta_{K, k}}, \\
\tan \left(\delta_{K, k}\right) & =-\frac{U \csc (k d)}{2 J_{K}} .
\end{aligned}
$$

In the limit of $U \rightarrow 0$, this reduces to the trivial solution $\psi_{K, k}\left(r_{i}\right)=\cos \left(k r_{i}\right)$ for two non-interacting bosons, while in the opposite limit of $U / J_{K} \rightarrow \pm \infty$, Eq. (13) yields the fermionized solution $\psi_{K, k}\left(r_{i}\right)=\sin \left(k\left|r_{i}\right|\right)$. At the edges of the scattering band $k \rightarrow 0, \pi / d$, we can define a generalized $1 \mathrm{D}$ scattering length $a_{K}$ via [5]

$$
a_{K}=-\lim _{k \rightarrow 0} \frac{\partial \delta_{K, k}}{\partial k}=-\frac{2 d J_{K}}{U},
$$

which is thus positive for $U<0$ and negative for $U>0$, as can be intuitively understood on the basis of perturbation theory [6]. Thus, in the case of $U<0$, a pair of co-localised particles (forming a dimer discussed below) has an energy below that of a pair of unbound particles, causing therefore an effective repulsion between the unbound particles by pushing their energy upwards. Conversely, the energy of the repulsively bound pair $U>0$ is above the energy of the unbound pair of particles, which lowers their energy causing an effective attraction.

(ii) The on-site interaction $U \neq 0$ can bind the two bosonic particles together into a close dimer [4, 5, 6] whose energy is above $(U>0)$ or below $(U<0)$ the continuum (11) of scattering states. We now derive simple analytic solutions for the bound dimer states. For $|K|=\pi / d\left(J_{K}=0\right)$, Eq. (10) immediately yields $E_{\pi / d}^{(2)}=U$ and

$$
\psi_{\pi / d}(0)=1, \quad \psi_{\pi / d}\left(r_{i} \neq 0\right)=0 .
$$

For $K \in(-\pi / d, \pi / d)$, using the exponential ansatz $\psi_{K}\left(r_{i}\right)=C \alpha_{K}^{|i|}$, where $C$ is a normalization constant, from Eq. (10) we have

$$
\begin{aligned}
-2 J_{K} \alpha_{K}+U & =E_{K}^{(2)}, \\
-J_{K} \frac{\alpha_{K}^{|i|-1}+\alpha_{K}^{|i|+1}}{\alpha_{K}^{|i|}} & =E_{K}^{(2)},
\end{aligned}
$$

where the unknowns are $E_{K}^{(2)}$ and $\alpha_{K}$. The solutions for $\alpha_{K}$ are given by

$$
\alpha_{K}=\mathcal{U}_{K} \pm \sqrt{\mathcal{U}_{K}^{2}+1}, \quad \mathcal{U}_{K} \equiv \frac{U}{2 J_{K}} .
$$



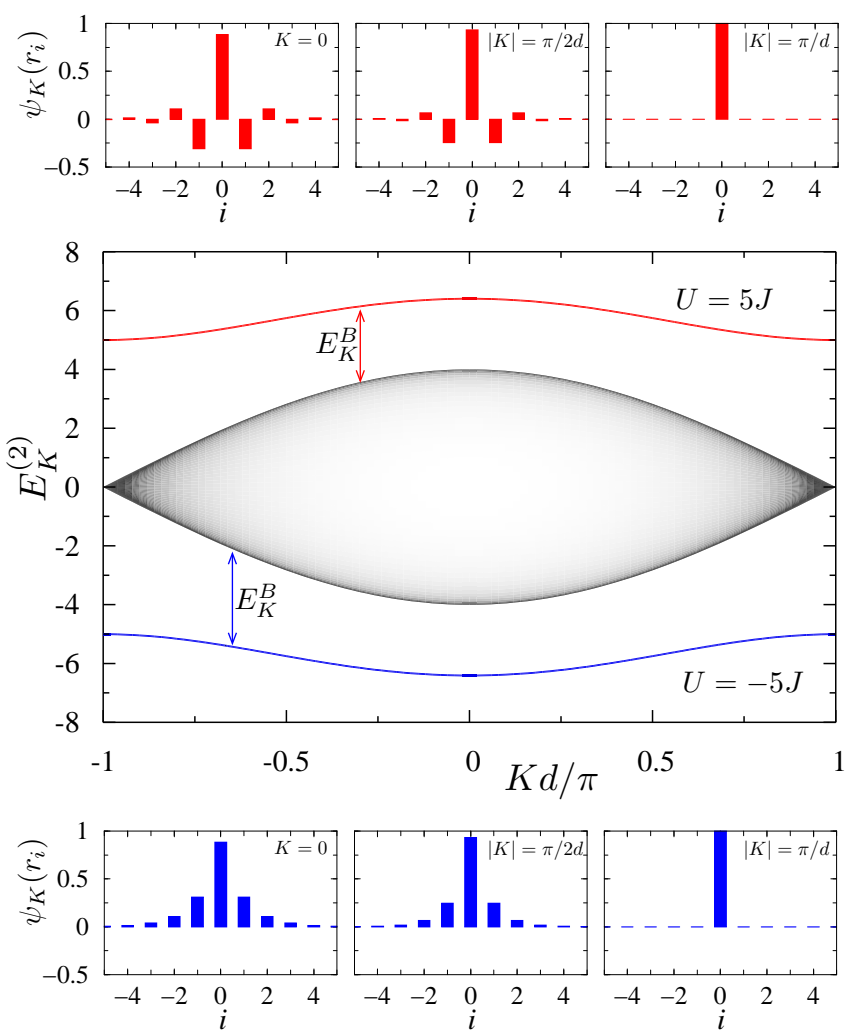

FIG. 1: Energies $E_{K}^{(2)}$ versus the center-of-mass momentum $K$ for a pair of bosons in a $1 \mathrm{D}$ lattice described by the Hubbard model. The continuum spectrum corresponds to energies (11) of the scattering states, with the shading proportional to the density of states (12). The (blue) line below and the (red) line above the scattering band are, respectively, the energies of the attractively-bound dimer with $U=-5 J$ and the repulsivelybound dimer with $U=5 J$. Their relative coordinate wavefunctions (17b) and (18b) at $|K|=0, \pi / 2 d, \pi / d$ are shown on the bottom and the top panels.

A normalizable bound state corresponds to $\left|\alpha_{K}\right|<1$, which exists for any $U \neq 0$. Thus, for attractive interaction, $U<0$, we have

$$
\alpha_{K}=\sqrt{\mathcal{U}_{K}^{2}+1}-\left|\mathcal{U}_{K}\right|>0,
$$

with which for the energy and normalized relative coordinate wavefunction we obtain

$$
\begin{aligned}
E_{K}^{(2)} & =-\sqrt{U^{2}+4 J_{K}^{2}}, \\
\psi_{K}\left(r_{i}\right) & =\frac{\sqrt{\left|\mathcal{U}_{K}\right|}}{\sqrt[4]{\mathcal{U}_{K}^{2}+1}}\left(\sqrt{\mathcal{U}_{K}^{2}+1}-\left|\mathcal{U}_{K}\right|\right)^{|i|} .
\end{aligned}
$$

The lowest energy of an attractively-bound dimer is $E_{0}^{(2)}=-\sqrt{U^{2}+16 J^{2}}$ attained at $K=0$, while the highest energy is $E_{\pi / d}^{(2)}=-|U|=U$ at $K= \pm \pi / d$. We can define the binding energy for a dimer with momentum $K$ as the difference between the energy of the bound pair $E_{K}^{(2)}$ and the energy at the bottom of the scattering band for an unbound pair $E_{K, 0}^{(2)}=-2 J_{K}$ (see Fig. 1, central panel),

$$
E_{K}^{B} \equiv E_{K}^{(2)}-E_{K, 0}^{(2)}=2 J_{K}-\sqrt{U^{2}+4 J_{K}^{2}}<0 .
$$

For repulsive interaction, $U>0$, we have

$$
\alpha_{K}=\mathcal{U}_{K}-\sqrt{\mathcal{U}_{K}^{2}+1}<0,
$$

and the dimer energy and wavefunction are given by

$$
\begin{aligned}
E_{K}^{(2)} & =\sqrt{U^{2}+4 J_{K}^{2}}, \\
\psi_{K}\left(r_{i}\right) & =\frac{\sqrt{\mathcal{U}_{K}}}{\sqrt[4]{\mathcal{U}_{K}^{2}+1}}\left(\mathcal{U}_{K}-\sqrt{\mathcal{U}_{K}^{2}+1}\right)^{|i|} .
\end{aligned}
$$

The lowest and highest energies of a repulsively-bound dimer are given by $E_{\pi / d}^{(2)}=|U|=U$ and $E_{0}^{(2)}=$ $\sqrt{U^{2}+16 J^{2}}$, attained, respectively, at $K= \pm \pi / d$ and $K=0$. The dimer binding energy, counted from the top of the scattering band $E_{K, \pi}^{(2)}=2 J_{K}$ (see Fig. 1), is now positive, given by

$$
E_{K}^{B} \equiv E_{K}^{(2)}-E_{K, \pi}^{(2)}=\sqrt{U^{2}+4 J_{K}^{2}}-2 J_{K}>0 .
$$

Clearly, for a given value of momentum $K$ of the dimer, the stronger the on-site interaction $|U|$, the smaller the extent of the dimer wavefunction. In the case of repulsive interaction $U>0\left(\alpha_{K}<0\right)$, the sign of wavefunction (18b) alternates between the neighboring sites $i$. Remarkably, when $|K|=\pi / d$, and thereby $J_{K}=$ 0 , the dimer wavefunction is completely localized at $r_{i}=0$ for any $U \neq 0$. To illustrate the foregoing discussion, in Fig. 1 we show the dispersion relations (17a) and (18a) and the wavefunctions (17b) and (18b) at $|K|=0, \pi / 2 d, \pi / d$ for attractively- and repulsivelybound dimers.

The dimer effective mass $M^{*}$ for small $K$ is given by

$$
M^{*}=\hbar^{2}\left[\frac{\partial^{2} E_{K}^{(2)}}{\partial K^{2}}\right]_{K=0}^{-1}= \pm \frac{\hbar^{2} \sqrt{U^{2}+(4 J)^{2}}}{4 d^{2} J^{2}},
$$

where the upper sign corresponds to attractive interaction $U<0$, leading to positive $M^{*}$; while the lower sign stands for repulsive interaction $U>0$, for which the effective mass $M^{*}$ is negative. For weak interaction $|U| \ll J$, we have $M^{*} \simeq \pm \hbar^{2} /\left(d^{2} J\right)= \pm 2 m^{*}$, i.e., twice the single particle effective mass $m^{*}$ of Eq. (5). On the other hand, for strong interaction $|U| \gg J$, we obtain $M^{*} \simeq \hbar^{2} /\left(2 d^{2} J^{(2)}\right)$, where $J^{(2)} \equiv-2 J^{2} / U$ is the effective tunnelling rate of the dimer between the neighboring lattice sites [4, 5, 6, 7]. Now the dimer effective mass is large due to its slow tunneling $\left|J^{(2)}\right| \ll J$, with the sign of $J^{(2)}$ determining the sign of $M^{*}$. In this limit the energies (17a) and (18a) can be approximated as

$$
E_{K}^{(2)} \simeq\left(U-2 J^{(2)}\right)-2 J^{(2)} \cos (K d),
$$


where the first term on the right-hand side represents the dimer "internal energy", while the second term is the kinetic energy of a dimer with quasimomentum $K$.

To summarize, we have considered a pair of bosonic particles in a $1 \mathrm{D}$ tight-binding periodic potential described by the Bose-Hubbard model and derived exact analytic solutions for the two-particle problem.
These solutions describe the scattering states as well as interaction-bound dimer states of the two particles. We have obtained simple explicit expressions for the energies and wavefunctions of the two-particle states and outlined their properties. Our results are relevant to the recent experimental [4] and theoretical [5, 6, 7] studies of strongly interacting pairs of atoms in an optical lattice.
[1] A.L. Fetter and J.D. Walecka, Quantum Theory of Many Particle Systems (McGraw-Hill, New York, 1971); N.J. Ashcroft and and N.D. Mermin, Solid State Physics (International Thomson Publishing, New York, 1976).

[2] O. Morsch and M. Oberthaler, Rev. Mod. Phys. 78, 179 (2006); I. Bloch, J. Phys. B: At. Mol. Opt. Phys. 38, S629 (2005); D. Jaksch and P. Zoller, Ann. Phys. (N.Y.) 315, $52(2005)$.

[3] D. Jaksch, C. Bruder, J.I. Cirac, C.W. Gardiner, and P. Zoller, Phys. Rev. Lett. 81, 3108 (1998); M. Greiner, O. Mandel, T. Esslinger, T.W. Hänsch, and I. Bloch, Nature 415, 39 (2002).
[4] K. Winkler, G. Thalhammer, F. Lang, R. Grimm, J. Hecker Denschlag, A.J. Daley, A. Kantian, H.P. Büchler, and P. Zoller, Nature 441, 853 (2006); J. Hecker Denschlag and A.J. Daley, arXiv:cond-mat/0610393.

[5] R. Piil and K. Mølmer, Phys. Rev. A 76, 023607 (2007); N. Nygaard, R. Piil, and K. Mølmer, arXiv:0802.3808.

[6] D. Petrosyan, B. Schmidt, J.R. Anglin, and M. Fleischhauer, Phys. Rev. A 76, 033606 (2007).

[7] M. Valiente and D. Petrosyan, Europhys. Lett. (in press); arXiv:0803.0617. 